\title{
Three-dimensional localization and optical imaging of objects in turbid media with independent component analysis
}

Min Xu

Fairfield University, mxu@fairfield.edu

M. Alrubaiee

S. K. Gaven

Eollow. Afhis and additional works at: https://digitalcommons.fairfield.edu/physics-facultypubs

(C) 2005 The Optical Society. This paper was published in Applied Optics and is made available as an electronic reprint with the permission of OSA. The paper can be found at the following URL on the OSA website: http://www.opticsinfobase.org/ao/abstract.cfm?URI=ao-44-10-1889. Systematic or multiple reproduction or distribution to multiple locations via electronic or other means is prohibited and is subject to penalties under law.

\section{Peer Reviewed}

\section{Repository Citation}

Xu, Min; Alrubaiee, M.; Gaven, S. K.; and Alfano, R. R., "Three-dimensional localization and optical imaging of objects in turbid media with independent component analysis" (2005). Physics Faculty Publications.

12.

https://digitalcommons.fairfield.edu/physics-facultypubs/12

\section{Published Citation}

M. Xu, M. Alrubaiee, S. K. Gayen, and R. R. Alfano, "Three-dimensional localization and optical imaging of objects in turbid media with independent component analysis," Applied Optics. 44(10), 1889-1897 (2005) http://www.opticsinfobase.org/ao/abstract.cfm?URI=ao-44-10-1889

This item has been accepted for inclusion in DigitalCommons@Fairfield by an authorized administrator of DigitalCommons@Fairfield. It is brought to you by DigitalCommons@Fairfield with permission from the rightsholder(s) and is protected by copyright and/or related rights. You are free to use this item in any way that is permitted by the copyright and related rights legislation that applies to your use. For other uses, you need to obtain permission from the rights-holder(s) directly, unless additional rights are indicated by a Creative Commons license in the record and/or on the work itself. For more information, please contact digitalcommons@fairfield.edu. 


\title{
Three-dimensional localization and optical imaging of objects in turbid media with independent component analysis
}

\author{
M. Xu, M. Alrubaiee, S. K. Gayen, and R. R. Alfano
}

\begin{abstract}
A new approach for optical imaging and localization of objects in turbid media that makes use of the independent component analysis (ICA) from information theory is demonstrated. Experimental arrangement realizes a multisource illumination of a turbid medium with embedded objects and a multidetector acquisition of transmitted light on the medium boundary. The resulting spatial diversity and multiple angular observations provide robust data for three-dimensional localization and characterization of absorbing and scattering inhomogeneities embedded in a turbid medium. ICA of the perturbations in the spatial intensity distribution on the medium boundary sorts out the embedded objects, and their locations are obtained from Green's function analysis based on any appropriate light propagation model. Imaging experiments were carried out on two highly scattering samples of thickness approximately 50 times the transport mean-free path of the respective medium. One turbid medium had two embedded absorptive objects, and the other had four scattering objects. An independent component separation of the signal, in conjunction with diffusive photon migration theory, was used to locate the embedded inhomogeneities. In both cases, improved lateral and axial localizations of the objects over the result obtained by use of common photon migration reconstruction algorithms were achieved. The approach is applicable to different medium geometries, can be used with any suitable photon propagation model, and is amenable to near-real-time imaging applications. () 2005 Optical Society of America
\end{abstract}

OCIS codes: $170.7050,170.5280,170.3660,100.3190,100.3010$.

\section{Introduction}

Optical tomographic imaging of objects in turbid media is an aggressively pursued area of contemporary research that derives impetus from a variety of potential practical applications. ${ }^{1-17}$ Of particular interest are medical applications in which optical tomography and spectroscopy have the potential to provide diagnostic information about tumors in breast and prostate tissue and functional information about brain activities. Simultaneous developments in experimental apparatus and techniques for object interrogation and signal acquisition, ,2,4,5,18,19 analytical models for light propagation, ${ }^{10,20-22}$ and computer al-

The authors are with the Department of Physics, the Institute for Ultrafast Spectroscopy and Lasers, New York State Center of Advanced Technology for Ultrafast Photonic Materials and Applications, City College of New York, 136th Street Convent Avenue J419, New York, New York 10031.

Received 14 July 2004; revised manuscript received 24 November 2004; accepted 5 December 2004.

0003-6935/05/101889-09\$15.00/0

(C) 2005 Optical Society of America gorithms for image reconstruction ${ }^{6,8}$ hold promise for realization of these potentials of optical tomography.

Researchers today use continuous-wave, amplitudemodulated, or ultrashort light pulses to probe the target(s) embedded in the turbid medium and obtain steady-state, frequency-domain, or time-varying optical signals, respectively, by using a variety of detection schemes. ${ }^{2,4,5,18,19}$ Multiple scattering of light in turbid media, such as breast tissue, precludes direct imaging of embedded targets. One then resorts to an inverse image reconstruction (IIR) ${ }^{6,8}$ approach that uses a forward model for light propagation, the measured light intensity distribution on the boundary of the turbid medium, and an inversion algorithm to generate a map of the optical properties, such as the absorption coefficient $\left(\mu_{a}\right)$ and the scattering coefficient $\left(\mu_{s}\right)$, of the medium and the embedded objects. The objects are desired to appear as localized inhomogeneities in the spatial distribution of these optical coefficients.

The inversion problem is ill posed, and its adequate theoretical treatment is critical to the achievement of a unique solution. ${ }^{8}$ Although three general approaches-Radon-transform-type straight line in- 
tegrals, ${ }^{23}$ modeling of light scattering as a Markov random process, ${ }^{24,25}$ and development and inversions of a partial differential equation (PDE) of diffusion type-are pursued, it is the PDE-based methods that seem more practical in consideration of the signal-tonoise $(\mathrm{S} / \mathrm{N})$ ratio of the data and computationally efficient methods available for solution. ${ }^{6,8}$ The commonly used PDE is the diffusion approximation (DA) of the radiative transfer equation (RTE). Both iterative reconstruction and noniterative linearized inversion approaches have been used to solve the inversion problem, which is weakly nonlinear with limited success. The reconstruction of images with adequate spatial resolution and optical contrast and the determination of the location of the inhomogeneities remain formidable tasks. The time required for data acquisition and image reconstruction is another important consideration.

In this article we present a simple and fast approach that employs (for test of concept) continuouswave transillumination measurements and a novel algorithm based on independent component analysis (ICA) ${ }^{26,27}$ from information theory to locate tumorlike inhomogeneities embedded in breast-simulating turbid media. ICA has been successfully applied in a variety of other applications. ${ }^{27-30}$ We refer to this information-theory-inspired approach as optical tomography that uses independent component analysis, abbreviated as OPTICA. Experimental arrangement for OPTICA realizes a multisource illumination and multidetector signal-acquisition scheme that provides a variety of spatial and angular views that are essential for three-dimensional (3-D) object localization. Multisource illumination is realized in practice by scanning the input surface (or source plane) across the incident beam in a two-dimensional (2-D) array of points $\left(x_{s k}, y_{s k} ; k=1,2, \ldots, n\right)$. Corresponding to illumination of the $k$ th grid point on the source plane, a charge-coupled device (CCD) camera records the spatial intensity distribution, $I_{k}\left(x_{d}, y_{d}\right)$, on the exit surface (or detector plane). Every pixel of the CCD camera thus acts as a detector implementing the multidetector measurement arrangement. The difference, $\Delta I_{k}\left(x_{d}, y_{d}\right)$, between the above-mentioned spatial intensity distribution, $I_{k}\left(x_{d}, y_{d}\right)$, and an estimated background (say, an averaged intensity distribution from different source scanning positions) provides the perturbation in the spatial intensity distribution in the detector plane for illumination at the $k$ th grid point.

The localization algorithm is based on the premise that each object (or inhomogeneity) within the turbid medium alters the propagation of light through the medium. Consequently, the spatial distribution of the light intensity at the detector plane of the medium is different with embedded inhomogeneities than that without them. The influence of an object on $\Delta I_{k}\left(x_{d}, y_{d}\right)$ involves propagation of light from the source to the object, and from the object to the detector, and can be described in terms of two Green's functions (propagators): The first $G\left(\mathbf{r}, \mathbf{r}_{s}\right)$ describes light propagation from the source at $\mathbf{r}_{s}$ to the object at $\mathbf{r}$, and the second
$G\left(\mathbf{r}_{d}, \mathbf{r}\right)$ describes that from the object to the detector at $\mathbf{r}_{d}$. To correlate the perturbations in the light intensity distributions, $\Delta I_{k}\left(x_{d}, y_{d}\right)$, with the objects embedded in the turbid medium, we assumed that these objects illuminated by the incident wave are virtual sources and that $\Delta I_{k}\left(x_{d}, y_{d}\right)$ are taken to be some weighted mixtures of signals arriving from these virtual sources to the detector plane. ICA assumes these virtual sources to be independent, and based on that assumption it provides the independent components. The number of leading independent components is the same as the number of the embedded objects. The effective contributions of independent components to the light intensity distribution on the source and detector planes are proportional to the projection of the Green's function, $G\left(\mathbf{r}, \mathbf{r}_{s}\right)$ and $G\left(\mathbf{r}_{d}, \mathbf{r}\right)$, on the source and detector planes, respectively. The location and characteristics of the objects are obtained from fitting either or both of these projections to those of the model Green's function in the background medium.

The remainder of the article is organized as follows. In Section 2, we present the general theoretical framework for OPTICA and then discuss the specific case of a turbid medium in the form of a slab. However, the approach can be adapted to any arbitrary geometry. Scattering and absorbing objects are considered separately. Section 3 presents the experimental methods, materials, and parameters. The results are presented in Section 4. Finally, the implications of these results and the scope of OPTICA are discussed in Section 5.

\section{Theoretical Formalism}

In the linearized scheme of inversion, the perturbation of the detected light intensities on the boundaries of the medium (the scattered wave field) due to absorptive and scattering objects (inhomogeneities) is given by ${ }^{3,13}$

$$
\begin{aligned}
\phi_{\mathrm{sca}}\left(\mathbf{r}_{d}, \mathbf{r}_{s}\right)= & -\int G\left(\mathbf{r}_{d}, \mathbf{r}\right) \delta \mu_{a}(\mathbf{r}) c G\left(\mathbf{r}, \mathbf{r}_{s}\right) d^{3} \mathbf{r} \\
& -\int \mathrm{d}^{3} \mathbf{r} \delta D(\mathbf{r}) c \nabla_{\mathbf{r}} G\left(\mathbf{r}_{d}, \mathbf{r}\right) \cdot \nabla_{\mathbf{r}} G\left(\mathbf{r}, \mathbf{r}_{s}\right)
\end{aligned}
$$

in the DA (Ref. 31) when illuminated by a unit point source, where $\mathbf{r}_{s}, \mathbf{r}$ and $\mathbf{r}_{d}$ are the positions of the source, the inhomogeneity, and the detector, respectively; $\delta \mu_{a}=\mu_{a, \text { obj }}-\mu_{a}$ and $\delta D=D_{\text {obj }}-D$ are the differences in the absorption coefficient and the diffusion coefficient, respectively, between the inhomogeneity and the background; $c$ is the speed of light in the medium; and $G\left(\mathbf{r}, \mathbf{r}^{\prime}\right)$ is the Green's function describing light propagation from $\mathbf{r}^{\prime}$ to $\mathbf{r}$ inside the background turbid medium of the absorption and diffusion coefficients $\mu_{a}$ and $D$, respectively. We do not explicitly include the modulation frequency $\omega$ of 
the incident wave in the arguments of Eq. (1) for clarity. The following formalism can be applied to continuous-wave, frequency-domain, and timeresolved measurements. The time-domain measurement is first Fourier transformed over time to obtain data over many different frequencies. Although Eq. (1) starts with DA, it should be emphasized that the formalism is not limited to DA, but can be used with other models of light propagation in turbid media, such as the cumulant approximation, $20,22,32$ the random-walk model, ${ }^{10,24}$ and radiative transfer ${ }^{17,33}$ when they are linearized.

The Green's function $G$ for a slab geometry in the diffusion approximation is given by

$$
\begin{aligned}
G\left(\mathbf{r}, \mathbf{r}^{\prime}\right) & \equiv G\left(\rho, z, z^{\prime}\right) \\
& =\frac{1}{4 \pi D} \sum_{k=-\infty}^{\infty}\left[\frac{\exp \left(-\kappa r_{k}{ }^{+}\right)}{r_{k}^{+}}-\frac{\exp \left(-\kappa r_{k}{ }^{-}\right)}{r_{k}{ }^{-}}\right], \\
r_{k}{ }^{ \pm} & =\left[\rho^{2}+\left(z \mp z^{\prime} \pm 2 k d\right)^{2}\right]^{1 / 2}
\end{aligned}
$$

for an incident amplitude-modulated wave of modulation frequency $\omega$, where $k=0, \pm 1, \pm 2, \ldots, \rho=$ $\left.\left[x-x^{\prime}\right)^{2}+\left(y-y^{\prime}\right)^{2}\right]^{1 / 2}$ is the distance between the two points $\mathbf{r}=(x, y, z)$ and $\mathbf{r}^{\prime}=\left(x^{\prime}, y^{\prime}, z^{\prime}\right)$ projected onto the $x y$ plane, $\kappa=\left[\left(\mu_{a}-i \omega / c\right) / D\right]^{1 / 2}$ chosen to have a nonnegative real part, and the extrapolated boundaries of the slab are located at $z=0$ and $z=d$ $=L_{z}+2 z_{e}$, respectively, where $L_{z}$ is the physical thickness of the slab and the extrapolation length $z_{e}$ should be determined from the boundary condition of the slab. ${ }^{34-36}$ Equation (2) serves as the model Green's function for the uniform background medium of a slab geometry. The modulation frequency $\omega$ $=0$ for continuous-wave light.

In practice, the projections of the Green's function on the source and detector planes are determined from the measured perturbations in the light intensity distribution through independent component analysis. The comparison with the Green's function computed with Eq. (2) is then used to locate and characterize the inhomogeneities. We develop the formalism for absorptive and scattering inhomogeneities in the Subsections 2.A and 2.B, respectively.

\section{A. Absorptive Inhomogeneity}

The assumption that absorptive inhomogeneities are localized [that is, the $j$ th one is contained in volume $V_{j}$ centered at $\mathbf{r}_{j}(1 \leq j \leq J)$ ] enables one to rewrite the scattered wave field in Eq. (1) as

$$
-\phi_{\mathrm{sca}}\left(\mathbf{r}_{d}, \mathbf{r}_{s}\right)=\sum_{j=1}^{J} G\left(\mathbf{r}_{d}, \mathbf{r}_{j}\right) q_{j} G\left(\mathbf{r}_{j}, \mathbf{r}_{s}\right),
$$

where $q_{j}=\delta \mu_{a}\left(\mathbf{r}_{j}\right) c V_{j}$ is the absorption strength of the $j$ th inhomogeneity. The scattered wave may be interpreted as an instantaneous linear mixture ${ }^{37}$

$$
\mathbf{x}\left(\mathbf{r}_{s}\right)=A \mathbf{s}\left(\mathbf{r}_{s}\right) .
$$

Here $\mathbf{s}\left(\mathbf{r}_{s}\right)=\left[q_{1} G\left(\mathbf{r}_{1}, \mathbf{r}_{s}\right), \ldots, q_{J} G\left(\mathbf{r}_{J}, \mathbf{r}_{s}\right)\right]^{T}$ represents the $J$ virtual sources; i.e., the $J$ inhomogeneities illuminated by the incident wave, $A$ is the mixing matrix given by

$$
A=\left[\begin{array}{cccc}
G\left(\mathbf{r}_{d_{1}}, \mathbf{r}_{1}\right) & G\left(\mathbf{r}_{d_{1}}, \mathbf{r}_{2}\right) & \ldots & G\left(\mathbf{r}_{d_{1}}, \mathbf{r}_{J}\right) \\
G\left(\mathbf{r}_{d_{2}}, \mathbf{r}_{1}\right) & G\left(\mathbf{r}_{d_{2}}, \mathbf{r}_{2}\right) & \ldots & G\left(\mathbf{r}_{d_{2}}, \mathbf{r}_{J}\right) \\
\vdots & \vdots & \ddots & \vdots \\
G\left(\mathbf{r}_{d_{m}}, \mathbf{r}_{1}\right) & G\left(\mathbf{r}_{d_{m}}, \mathbf{r}_{2}\right) & \ldots & G\left(\mathbf{r}_{d_{m}}, \mathbf{r}_{J}\right)
\end{array}\right]
$$

whose $j$ th column (mixing vector) provides the weight factors for the contributions from the $j$ th inhomogeneity to the detectors, and $\mathbf{x}\left(\mathbf{r}_{s}\right)=$ $\left[-\phi_{\text {sca }}\left(\mathbf{r}_{d_{1}}, \mathbf{r}_{s}\right), \ldots,-\phi_{\text {sca }}\left(\mathbf{r}_{d_{m}}, \mathbf{r}_{s}\right)\right]^{T}$ is the observed light intensity change. The superscript $T$ denotes transposition. The incident light source scans a total of $n$ positions $\mathbf{r}_{s_{1}}, \ldots, \mathbf{r}_{s_{n}}$ sequentially. For each source position $\mathbf{r}_{s_{j}}$, the observation is made over $m$ positions $\mathbf{r}_{d_{1}}, \ldots, \mathbf{r}_{d_{m}}$. Each set of such measurement is considered data at one temporal sampling point, as used in the conventional instantaneous linear mixture model. ${ }^{38}$ The multisource, multidetector data set $\mathbf{x}\left(\mathbf{r}_{s}\right)$ thus describes signals observed in $m$ channels ( $m$ detectors) from $J$ virtual sources (or $J$ inhomogeneities) simultaneously over $n$ discrete temporal points ( $n$ spatial scanning points). One absorptive inhomogeneity is represented by one virtual source $q_{j} G\left(\mathbf{r}_{j}, \mathbf{r}_{s}\right)$. The virtual source $q_{j} G\left(\mathbf{r}_{j}, \mathbf{r}_{s}\right)$ represents the individual inhomogeneity illuminated by the incident wave and is similar to the concept of the secondary source in Huygen's principle. ${ }^{39}$ The role of detectors and sources can be interchanged owing to the reciprocal property of light propagation.

The principal assumption of this formalism is that the virtual source $q_{j} G\left(\mathbf{r}_{j}, \mathbf{r}_{s}\right)$ at the $j$ th inhomogeneity is independent of the virtual sources at other locations. Under this assumption, ICA can be used with the observations from the light source scanned at $n \gg J$ positions to separate out both virtual sources $\mathbf{s}\left(\mathbf{r}_{s}\right)$ and the mixing matrix $A .{ }^{26,37}$

ICA is a statistical approach to separate independent sources from linear instantaneous or convolutive mixtures of independent signals without relying on any specific knowledge of the sources except that they are independent. The sources are recovered by a minimization of a measure of dependence, such as mutual information, ${ }^{26,27}$ between the reconstructed sources. ${ }^{30,37}$ The recovered virtual sources and mixing vectors from ICA are unique up to permutation and scaling. ${ }^{30,37}$

The two Green's functions of light propagating from the source to the inhomogeneity and from the inhomogeneity to the detector are retrieved from the separated virtual sources $\mathbf{s}\left(\mathbf{r}_{s}\right)$ and the mixing matrix $A$. The $j$ th element $s_{j}\left(\mathbf{r}_{s}\right)$ of the virtual source array and the $j$ th column $\mathbf{a}_{j}$ (mixing vector) of the mixing matrix $A$ provide the scaled projections of the Green's function on the source and detector planes, $G\left(\mathbf{r}_{j}, \mathbf{r}_{s}\right)$ and $G\left(\mathbf{r}_{d}, \mathbf{r}_{j}\right)$, respectively. We can write 


$$
\begin{array}{r}
s_{j}\left(\mathbf{r}_{s}\right)=\alpha_{j} G\left(\mathbf{r}_{j}, \mathbf{r}_{s}\right), \\
a_{j}\left(\mathbf{r}_{d}\right)=\beta_{j} G\left(\mathbf{r}_{d}, \mathbf{r}_{j}\right),
\end{array}
$$

where $\alpha_{j}$ and $\beta_{j}$ are scaling constants for the $j$ th inhomogeneity.

Both the location and the strength of the jth object can be computed by a simple fitting procedure by use of Eq. (6). We adopted a least-square fitting procedure given by

$$
\begin{aligned}
& \min _{\mathbf{r}_{j}, \alpha_{j}, \beta_{j}}\left\{\sum_{\mathbf{r}_{s}}\left[\alpha_{j}{ }^{-1} s_{j}\left(\mathbf{r}_{s}\right)-G\left(\mathbf{r}_{j}, \mathbf{r}_{s}\right)\right]^{2}+\sum_{\mathbf{r}_{d}}\right. {\left[\beta_{j}^{-1} a_{j}\left(\mathbf{r}_{d}\right)\right.} \\
&\left.\left.-G\left(\mathbf{r}_{d}, \mathbf{r}_{j}\right)\right]^{2}\right\} .
\end{aligned}
$$

The fitting yields the location $\mathbf{r}_{j}$ of and the two scaling constants $\alpha_{j}$ and $\beta_{j}$ for the $j$ th inhomogeneity, whose absorption strength is then given by $q_{j}=\alpha_{j} \beta_{j}$.

\section{B. Scattering Inhomogeneity}

For scattering inhomogeneities, under the assumption that the inhomogeneities are localized in a few regions, the same analysis can be carried out as that for absorptive inhomogeneities. The only modification is that up to three virtual sources may appear for one scattering inhomogeneity corresponding to the $x, y, z$ components in the dot product $\nabla_{\mathbf{r}} G\left(\mathbf{r}_{d}, \mathbf{r}\right)$. $\nabla_{\mathbf{r}} G\left(\mathbf{r}, \mathbf{r}_{s}\right)=\partial_{x} G\left(\mathbf{r}_{d}, \mathbf{r}\right) \partial_{x} G\left(\mathbf{r}, \mathbf{r}_{s}\right)+\partial_{y} G\left(\mathbf{r}_{d}, \mathbf{r}\right) \partial_{y} G\left(\mathbf{r}, \mathbf{r}_{s}\right)$ $+\partial_{z} G\left(\mathbf{r}_{d}, \mathbf{r}\right) \partial_{z} G\left(\mathbf{r}, \mathbf{r}_{s}\right)$ in Eq. (1).

Introducing two auxiliary functions

$$
\begin{aligned}
g_{\perp}\left(\mathbf{r}, \mathbf{r}^{\prime}\right)= & \frac{1}{4 \pi D} \sum_{k=-\infty}^{+\infty}\left[\left(\kappa r_{k}^{+}+1\right) \frac{\exp \left(-\kappa r_{k}^{+}\right)}{\left(r_{k}^{+}\right)^{3}}\right. \\
& \left.-\left(\kappa r_{k}^{-}+1\right) \frac{\exp \left(-\kappa r_{k}^{-}\right)}{\left(r_{k}^{-}\right)^{3}}\right], \\
g_{z}\left(\mathbf{r}, \mathbf{r}^{\prime}\right)= & \frac{1}{4 \pi D} \sum_{k=-\infty}^{+\infty}\left\{\left(z-z^{\prime}+2 k d\right)\left(\kappa r_{k}^{+}+1\right)\right. \\
& \times \frac{\exp \left(-\kappa r_{k}^{+}\right)}{\left(r_{k}^{+}\right)^{3}}-\left(z+z^{\prime}-2 k d\right) \\
& \left.\times\left(\kappa r_{k}^{-}+1\right) \frac{\exp \left(-\kappa r_{k}^{-}\right)}{\left(r_{k}^{-}\right)^{3}}\right\},
\end{aligned}
$$

and the scattered wave due to scattering inhomogeneities can be rewritten as

$$
\begin{aligned}
\phi_{\mathrm{sca}}\left(\mathbf{r}_{d}, \mathbf{r}_{s}\right)= & -\int \mathrm{d}^{3} \mathbf{r} \delta D(\mathbf{r}) c\left\{\left[\left(x-x_{d}\right)\left(x-x_{s}\right)\right.\right. \\
& \left.+\left(y-y_{d}\right)\left(y-y_{s}\right)\right] g_{\perp}\left(\mathbf{r}, \mathbf{r}_{d}\right) g_{\perp}\left(\mathbf{r}, \mathbf{r}_{s}\right) \\
& \left.+g_{z}\left(\mathbf{r}, \mathbf{r}_{d}\right) g_{z}\left(\mathbf{r}, \mathbf{r}_{s}\right)\right\} .
\end{aligned}
$$

Denoting the scattering inhomogeneities as $q_{j}^{\prime}$ $=\delta D\left(\mathbf{r}_{j}\right) c V_{j}^{\prime}$, where $c$ is the speed of light in the medium, and $V_{j}^{\prime}$ is the volume of the $j$ th scattering inhomogeneity, the scattered wave field can be transformed to

$$
\begin{aligned}
-\phi_{\mathrm{sca}}\left(\mathbf{r}_{d}, \mathbf{r}_{s}\right)= & \sum_{j=1}^{J^{\prime}} g_{z}\left(\mathbf{r}_{j}, \mathbf{r}_{d}\right) q_{j}{ }^{\prime} g_{z}\left(\mathbf{r}_{j}, \mathbf{r}_{s}\right)+\sum_{j=1}^{J^{\prime}} \rho_{d j} \\
& \times \cos \theta_{d} g_{\perp}\left(\mathbf{r}_{j}, \mathbf{r}_{d}\right) q_{j}{ }^{\prime} \rho_{s j} \cos \theta_{s} g_{\perp}\left(\mathbf{r}_{j}, \mathbf{r}_{s}\right) \\
& +\sum_{j=1}^{J^{\prime}} \rho_{d j} \sin \theta_{d} g_{\perp}\left(\mathbf{r}_{j}, \mathbf{r}_{d}\right) q_{j}{ }^{\prime} \rho_{s j} \\
& \times \sin \theta_{s} g_{\perp}\left(\mathbf{r}_{j}, \mathbf{r}_{s}\right),
\end{aligned}
$$

where $\rho_{d j}=\left[\left(x_{d}-x_{j}\right)^{2}+\left(y_{d}-y_{j}\right)^{2}\right]^{1 / 2}, \rho_{s j}=\left[\left(x_{s}\right.\right.$ $\left.\left.-x_{j}\right)^{2}+\left(y_{s}-y_{j}\right)^{2}\right]^{1 / 2}$, and $\theta_{d}$ and $\theta_{s}$ are the azimuthal angles of $\mathbf{r}_{d}-\mathbf{r}_{j}$ and $\mathbf{r}_{s}-\mathbf{r}_{j}$, respectively. This scattered wave can be regarded as a mixture of contributions from $\left(3 J^{\prime}\right)$ virtual sources:

$$
\begin{array}{r}
q_{j}{ }^{\prime} g_{z}\left(\mathbf{r}_{j}, \mathbf{r}_{s}\right), \quad q_{j}{ }^{\prime} \rho_{s j} \cos \theta_{s} g_{\perp}\left(\mathbf{r}_{j}, \mathbf{r}_{s}\right), \\
q_{j}{ }^{\prime} \rho_{s j} \sin \theta_{s} g_{\perp}\left(\mathbf{r}_{j}, \mathbf{r}_{s}\right),
\end{array}
$$

with mixing vectors

$$
g_{z}\left(\mathbf{r}_{j}, \mathbf{r}_{d}\right), \quad \rho_{d j} \cos \theta_{d} g_{\perp}\left(\mathbf{r}_{j}, \mathbf{r}_{d}\right), \quad \rho_{d j} \sin \theta_{d} g_{\perp}\left(\mathbf{r}_{j}, \mathbf{r}_{d}\right),
$$

where $1 \leq j \leq J^{\prime}$, respectively. There are in general three virtual sources of specific patterns (one centrosymmetric and two dumbbell shaped) associated with one scattering inhomogeneity, whereas only one centrosymmetric virtual source is associated with one absorptive inhomogeneity. This difference may be used to discriminate absorptive inhomogeneities from scattering inhomogeneities. However, for scattering inhomogeneities deep within turbid media, only the $q_{j}^{\prime} g_{z}\left(\mathbf{r}_{j}, \mathbf{r}_{s}\right)$ virtual source remains significant and the other two are much diminished. In such a situation, other corroborative evidence, such as multiwavelength measurements, are required to determine the nature of inhomogeneities. Both the location and the strength of the jth scattering object are computed by fitting the retrieved virtual sources and mixing vectors to expressions (12) and (13), respectively.

No specific light propagation model is assumed in ICA. The only assumption is that virtual sources are mutually independent. The number of inhomogeneities within the medium is determined by the number of the independent components presented in the multisource, multidetector data set. The analysis of retrieved independent components from ICA then localizes and characterizes the absorptive and scattering inhomogeneities inside the turbid medium in which an appropriate model of the light propagator is adopted. When the noise level is high or systematic errors are present, or both, extra independent components may appear. Only the leading independent components need to be analyzed to detect and characterize the inhomogeneities of interest, and other components can be discarded. 


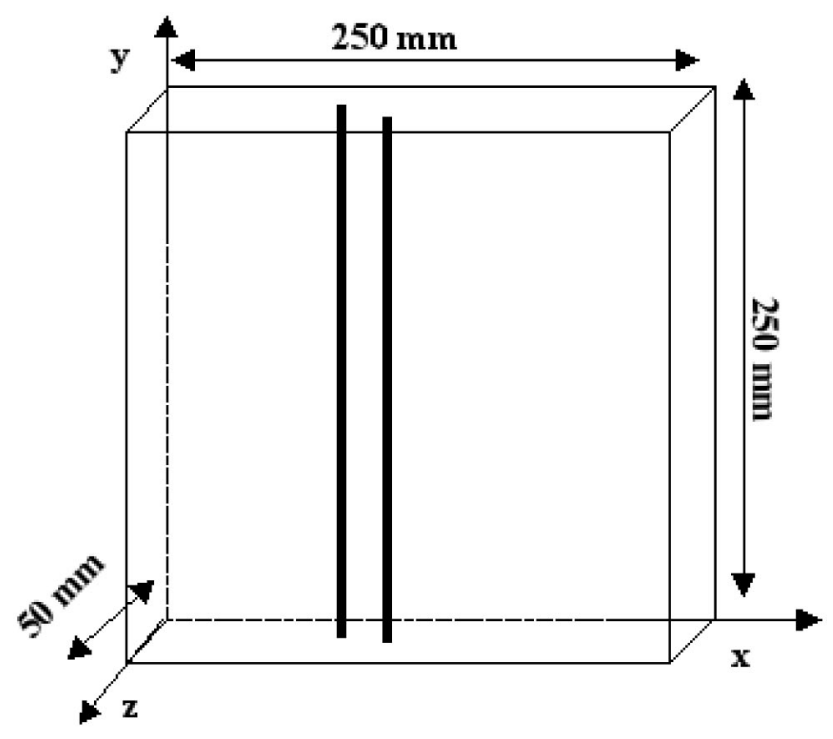

Fig. 1. Schematic diagram of specimen 1 comprising an Intralipid-10\% suspension in water with two long cylindrical absorbing objects of absorption coefficient $0.23 \mathrm{~mm}^{-1}$.

\section{Experimental Methods and Materials}

Two tissue-simulating phantoms with absorption and scattering coefficients within the reported range of values for healthy human breast tissue were used in the study reported here..$^{40}$

The first specimen (1), shown schematically in Fig. 1 , was a $250 \mathrm{~mm} \times 250 \mathrm{~mm} \times 50 \mathrm{~mm}$ transparent plastic container filled with Intralipid-10\% suspension in water. The concentration of Intralipid$10 \%$ was adjusted ${ }^{41}$ to provide a transport length $l_{t}$ $\sim 1 \mathrm{~mm}$ and an absorption coefficient $\mu_{a}=$ $0.003 \mathrm{~mm}^{-1}$ at $785 \mathrm{~nm}$, emulating those of human breast tissue. Two cylindrical glass tubes (outer diameter, $8 \mathrm{~mm}$; inner diameter, $6.98 \mathrm{~mm}$; and length, $250 \mathrm{~mm}$ ) were filled with an Intralipid-10\% suspension to provide the same scattering coefficient, but the absorption coefficient was changed to $0.023 \mathrm{~mm}^{-1}$ by the addition of absorbing ink. The two absorptive rods are placed at $(x, z)=(24,29) \mathrm{mm}$ and $(x, z)$ $=(47,33) \mathrm{mm}$, respectively, with the axes of cylindrical tubes along $y$.

The second specimen (2), loaned to us by J. C. Hebden of University College London and displayed schematically in Fig. 2, was a 166-mm-long, 82-mmwide, and 55-mm-thick slab made of materials with a reduced scattering coefficient $\mu_{s}{ }^{\prime} \sim 0.9 \mathrm{~mm}^{-1}$ (transport length, $l_{t} \sim 1.1 \mathrm{~mm}$ ) and an absorption coefficient $\mu_{a} \sim 0.006 \mathrm{~mm}^{-1}$. The slab contained four $5^{-}$ $\mathrm{mm}$-diameter, 5-mm-long cylindrical inhomogeneities. The center of each cylinder was located in the plane halfway between the front and the back faces of the slabs. The absorption coefficient of each cylinder was $0.006 \mathrm{~mm}^{-1}$, the same as that of the material of the slab, but the scattering coefficients were $4,2,1.5$, and 1.1 times greater. The first and the third cylinders, and the second and the fourth cylinders, are on two horizontal lines approximately

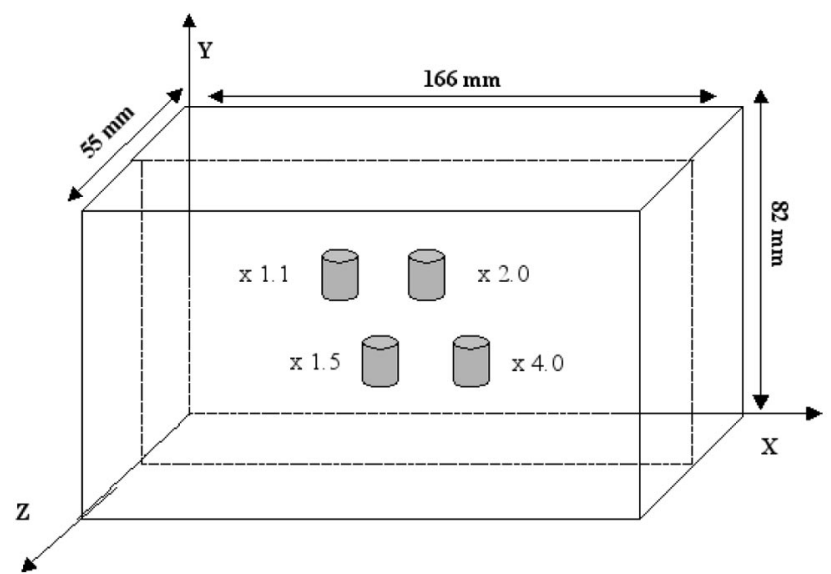

Fig. 2. Schematic diagram of specimen 2 obtained from University College London. It is a solid rectangular block embedded with four 5-mm-diameter and 5-mm-long scattering cylindrical objects with their centers on the central plane. The absorption and scattering characteristics of the specimens and the lateral positions of the four cylinders are described in the text.

$22 \mathrm{~mm}$ apart. The distance between neighboring cylinders is $11 \mathrm{~mm}$. Further details about the slab may be obtained from an article published by Hall et al. 42

The experimental arrangement used for imaging of these two specimens ( 1 and 2 ) is shown schematically in Fig. 3. For cw measurements, a $200-\mu \mathrm{m}$ fiber delivered a beam of 784-nm light from a diode laser (Ocean Optics R-2000) to illuminate the input surface (or source plane) of the specimen. A cooled CCD camera set at an acquisition time of $150 \mathrm{~ms}$ recorded 2-D intensity patterns of the light transmitted through the opposite side of the slab specimen. For time-resolved measurements, we used a 1-mm-diameter collimated beam of 784-nm, 150-fs, $1-\mathrm{kHz}$ repetition rate light pulses from a Ti:sapphire laser and amplifier system ${ }^{43}$ for sample illu-

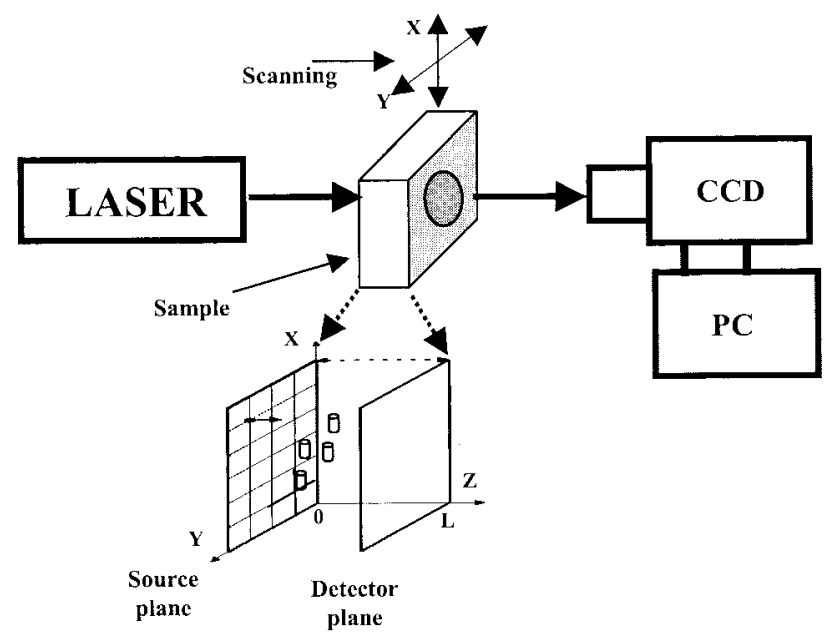

Fig. 3. Schematic diagram of the experimental arrangement for imaging objects embedded in a turbid medium. Inset shows the 2-D array in the input plane that is scanned across the incident laser beam. 
mination. An ultrafast gated intensified camera system (UGICS), which provided a FWHM gate width variable from $80 \mathrm{ps}$ to $6 \mathrm{~ns}$, recorded the $2-\mathrm{D}$ intensity patterns of the light transmitted through the opposite side of the slab. Computer-controlled $x y$ translation stages scanned the specimens in an array of points in the $x y$ plane, as displayed in Fig. 3. For the long cylindrical tubes in specimen 1, a line scan of 16 points with a step size of $2.5 \mathrm{~mm}$ along the $x$ axis was enough to obtain the $(x, z)$ locations of the absorbing cylinders. An array of $20 \times 18$ points with a step size of $2.5 \mathrm{~mm}$ across the lateral positions of the four inhomogeneities in specimen 2 was scanned to obtain their locations.

\section{Results}

Temporal profiles of the transmitted pulses were measured by use of the UGICS in the scan mode with an 80-ps gate width. The average optical properties of the turbid medium were estimated by fitting the temporal profiles to the DA of the RTE for a slab geometry.

ICA of the perturbations in the spatial intensity distributions provided the corresponding independent intensity distributions on the source and detector planes. ICA-generated independent intensity distributions on the source and detector planes are shown in the first and second rows, respectively, of Fig. 4 for the two absorbing cylinders in specimen 1 . The locations of the absorbing cylinders are obtained from fitting these independent component intensity distributions to those of the DA in a slab [Eq. (2)]. The first cylinder is found at $x=24 \mathrm{~mm}$, $29 \mathrm{~mm}$ away from the source plane and $21 \mathrm{~mm}$ away from the detector plane. The second cylinder is found at $x=47 \mathrm{~mm}, 33 \mathrm{~mm}$ away from the source plane and $17 \mathrm{~mm}$ away from the detector plane. The $(x, z)$ coordinates of both the cylinders agree to within $0.5 \mathrm{~mm}$ of their known locations. The absorption strengths of the two rods are estimated by use of a least-square fitting procedure [Eq. (7)]. The resolved absorption strengths are $q_{1}=0.152 \mathrm{~mm}^{2} / \mathrm{ps}$ and $q_{2}$ $=0.132 \mathrm{~mm}^{2} / \mathrm{ps}$, respectively, for the left and right rods. The values are $88 \%$ and $76 \%$, respectively, of the true value of $q=0.173 \mathrm{~mm}^{2} / \mathrm{ps}$.

The independent intensity distributions at the detector plane corresponding to the four scattering inhomogeneities in specimen 2 are displayed in Figs. 5(a)$5(\mathrm{~d})$. These independent components are then used to obtain the projections of the inhomogeneity detector Green's function, $G\left(\mathbf{r}_{d}, \mathbf{r}_{j}\right), j=1,2,3,4$, on the detector plane for the four small cylindrical scattering inhomogeneities embedded in specimen 2. The locations of the inhomogeneities are determined by fitting the projections to those of the model Green's function. The locations of all four inhomogeneities were obtained. Even the weakest scatterer, with a scattering coefficient just 1.1 times the background and hence considered to be rather unlikely to be found, 42 was detected. Positions along the $z$ axis (depth) of the cylinders were found to be at 28.1, 27.9, 27.1, and $32.6 \mathrm{~mm}$. Except for the last cylinder, the
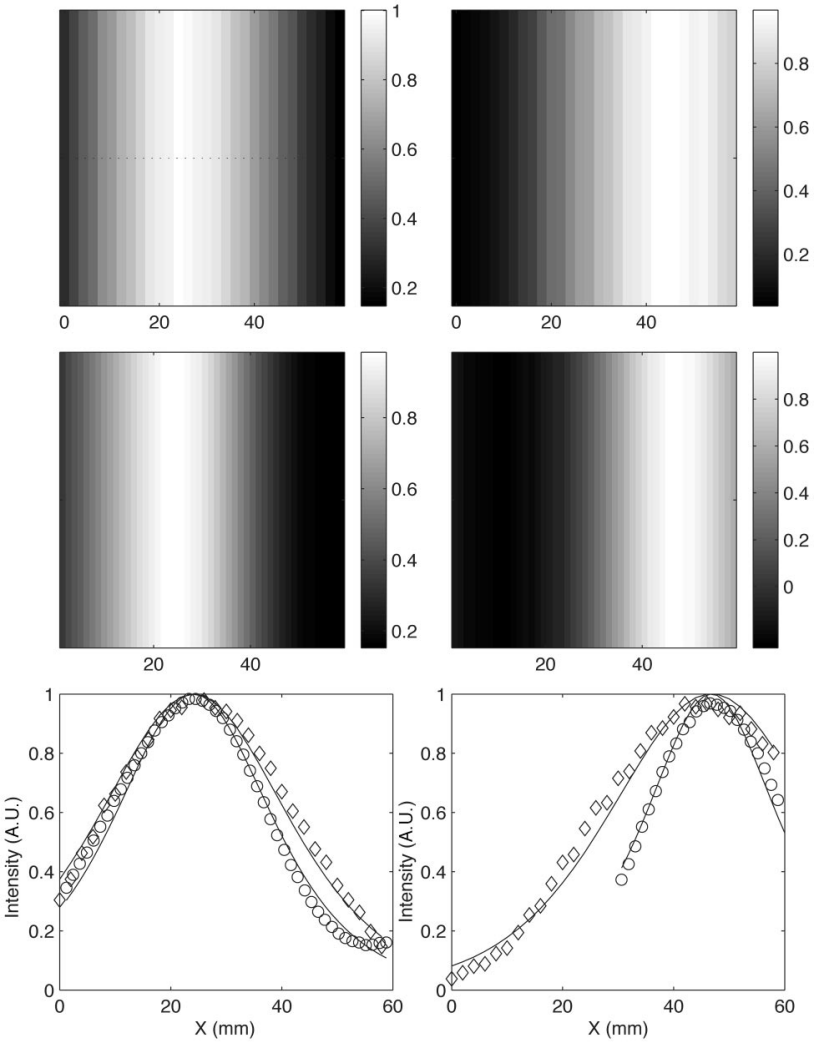

Fig. 4. Normalized independent spatial intensity distributions as a function of the lateral position $x$ at the input (or source) plane (first row) and the exit (or detector) plane (second row) generated by ICA for the two absorbing cylinders in specimen 1 . The horizontal profile of the intensity distributions on the source plane (diamond) and on the detector plane (circle) are displayed in the third row. Solid curves show the respective Green's-function fit used for obtaining the locations of the objects.

depth of the cylinders agree well with their known center positions of $27.5 \mathrm{~mm}$. The lateral positions are determined to be $(62,63),(48,33),(33,62)$, and $(18,33) \mathrm{mm}$ for the four scattering cylinders (see Table 1). The strongest and the third-strongest scatterers are on the same horizontal line $y \sim 62 \mathrm{~mm}$, whereas the second-strongest and the weakest scatterers are on the horizontal line $y \sim 33 \mathrm{~mm}$ with a spacing of $29 \mathrm{~mm}$. The four scatterers are separated by equal spacing, $\sim 14 \mathrm{~mm}$ in the horizontal direction. The lateral positions agree well with the known $(x, y)$ coordinate values. The uncertainties in location and separation are not greater than $3 \mathrm{~mm}$ except for the weakest target.

\section{Discussion}

The OPTICA presented in this article introduces the information theory technique of ICA to the problem of optical tomographic imaging of objects in turbid media. It is shown to provide object locations accurate to $\sim 1 \mathrm{~mm}$ in human-breast-like turbid media. It uses multiple-source (realized in this case through scanning of the sample in the $x y$ plane across the incident beam propagating in the $z$ direction) illumination and a multiple-detector (each pixel on the CCD may be 

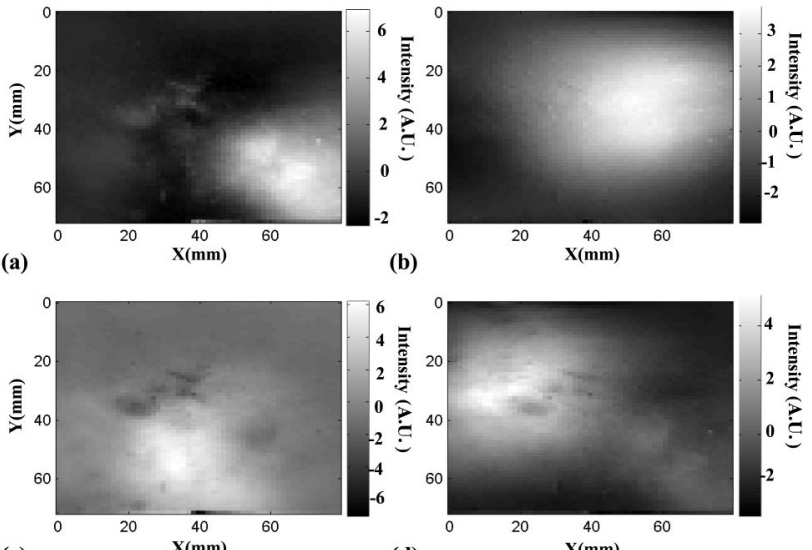

(c)

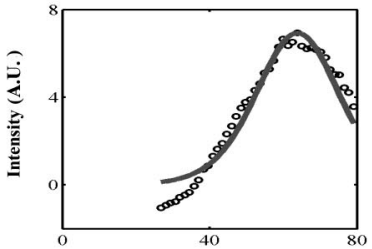

(e)

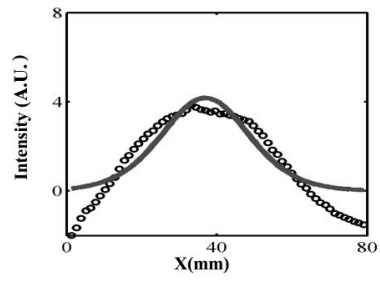

(d)
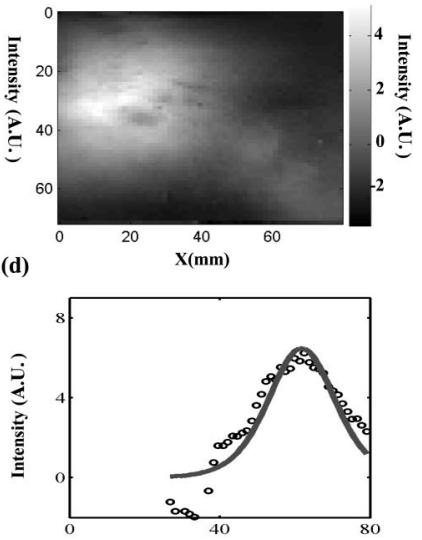

(f)

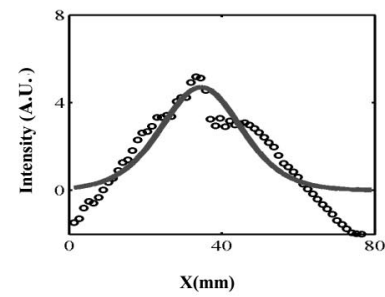

(h)

processing of the data and does not have to resort to any specific light propagation model for obtaining this information. Specific light propagation models are needed only in the later stage to determine the location by curve fitting of the Green's functions. OPTICA is not model specific; any appropriate model for light propagation, including the DA and the cumulant solutions of the RTE, may be used. OPTICA can be used with contrast agents such as fluorescence-based optical tomography as well.

Although we used the slab geometry in the study reported in this article, the approach does not depend on any specific geometry. It may be used for other geometries or even an arbitrary-shaped boundary. The approach is fast and is expected to be amenable to near-real-time detection and localization of objects in a turbid medium, which is a key consideration for in vivo medical imaging. The approach is remarkably sensitive, considering that it could discern all four cylinders in specimen 2. The approach successfully detected even the lowest-contrast inhomogeneity of the four that had a reduced scattering coefficient only $10 \%$ higher than the surrounding medium and was considered improbable to be detected. ${ }^{42}$ OPTICA obtains locations of the objects by fitting either or both of the Green's functions $G\left(\mathbf{r}, \mathbf{r}_{s}\right)$ and $G\left(\mathbf{r}_{d}, \mathbf{r}\right)$, and is suited for physically small inhomogeneities. Given its capability of identifying low-contrast small objects, the approach is expected to be useful for detecting tumors at their early stages of development, a coveted goal in medical imaging.

As demonstrated with specimen 1 and specimen 2, the approach could locate both absorptive and scattering objects. When both absorptive and scattering objects are present in the same turbid specimen, OPTICA can locate them, but their identification as absorbing or scattering entities becomes a more challenging task. As discussed in connection with expressions (12) and (13), each scattering inhomogeneity is expected to be represented by three virtual sources, yielding three pairs of effective intensity distributions each on the detector and source planes. When the background scattering is not severe and the $\mathrm{S} / \mathrm{N}$ ratio is high, contributions from all three virtual sources may be distinguished and the corresponding object may be identified as a scattering entity. Our simulation results support this assertion. For highly scattering conditions with lower $\mathrm{S} / \mathrm{N}$ ratios, contributions from the two dumbbell-shaped virtual sources may not be discerned, and corroborative information obtained by other means, such as measurements using light of different wavelengths, are required for identification of the object as an absorptive or scattering entity. Multiwavelength spectroscopic imaging measurements have the potential to provide diagnostic information, such as whether a tumor is malignant or benign.

In summary, OPTICA has the potential to emerge as a new versatile tool for locating targets in turbid media, particularly in diagnostic medical imaging and underwater imaging.

${ }^{a}$ Target strength is the ratio of the scattering coefficients of the target to that of the surrounding medium. The errors in location are not greater than $3 \mathrm{~mm}$. 
This study is supported in part by the U.S. Army Medical Research and Materials Command, Office of Naval Research, New York State Office of Science, Technology and Academic Research, and City University of New York organized research programs. M. $\mathrm{Xu}$ acknowledges the support by the Department of Army (grant DAMD17-02-1-0516). M. Alrubaiee thanks the National Science Foundation for an Advance Placement Fellowship. We acknowledge J. C. Hebden of University College London for the loan of specimen 2 and W. Cai for helpful discussions.

\section{References}

1. G. Muller, R. R. Alfano, S. R. Arridge, J. Beuthan, E. Gratton, M. Kaschke, B. R. Masters, S. Svanberg, and P. van der Zee, eds., Medical Optical Tomography: Functional Imaging and Monitoring, Vol. IS11 of SPIE Institute Series (SPIE, Bellingham, Wash., 1993).

2. A. Yodh and B. Chance, "Spectroscopy and imaging with diffusing light," Phys. Today 48, 38-40 (1995).

3. M. A. O'Leary, D. A. Boas, B. Chance, and A. G. Yodh, "Experimental images of heterogeneous turbid media by frequency-domain diffusing-photon tomography," Opt. Lett. 20, 426-428 (1995).

4. S. K. Gayen and R. R. Alfano, "Emerging optical biomedical imaging techniques," Opt. Photon. News 7, 17-22 (1996).

5. J. C. Hebden, S. R. Arridge, and D. T. Delpy, "Optical imaging in medicine: I. Experimental techniques,” Phys. Med. Biol. 42, 825-840 (1997).

6. S. R. Arridge and J. C. Hebden, "Optical imaging in medicine: II. Modelling and reconstruction," Phys. Med. Biol. 42, 841853 (1997).

7. W. Cai, S. K. Gayen, M. Xu, M. Zevallos, M. Alrubaiee, M. Lax, and R. R. Alfano, "Optical tomographic image reconstruction from ultrafast time-sliced transmission measurements," Appl. Opt. 38, 4237-4246 (1999).

8. S. R. Arridge, "Optical tomography in medical imaging," Inverse Probl. 15, R41-R93 (1999).

9. D. Grosenick, H. Wabnitz, H. H. Rinneberg, K. T. Moesta, and P. M. Schlag, "Development of a time-domain optical mammograph and first in vivo applications," Appl. Opt. 38, 2927-2943 (1999).

10. V. Chernomordik, D. Hattery, A. H. Gandjbakhche, A. Pifferi, P. Taroni, A. Torricelli, G. Valentini, and R. Cubeddu, "Quantification by random walk of the optical parameters of nonlocalized abnormalities embedded within tissuelike phantoms," Opt. Lett. 25, 951-953 (2000).

11. V. A. Markel and J. C. Schotland, "Inverse scattering for the diffusion equation with general boundary conditions," Phys. Rev. E 64, 035601 (2001).

12. A. H. Hielscher and S. Bartel, "Use of penalty terms in gradient-based iterative reconstruction schemes for optical tomography," J. Biomed. Opt. 6, 183-192 (2001).

13. M. Xu, M. Lax, and R. R. Alfano, "Time-resolved Fourier optical diffuse tomography," J. Opt. Soc. Am. A 18, 1535-1542 (2001).

14. B. A. Brooksby, H. Dehghani, B. W. Pogue, and K. D. Paulsen, "Near-infrared (NIR) tomography breast image reconstruction with a priori structural information from MRI: algorithm development for reconstructing heterogeneities," IEEE J. Sel. Top. Quantum Electron. 9, 199-209 (2003).

15. H. Dehghani, B. W. Pogue, S. P. Poplack, and K. D. Paulsen, "Multiwavelength three-dimensional near-infrared tomography of the breast: initial simulation, phantom, and clinical results," Appl. Opt. 42, 135-145 (2003).

16. J. C. Hebden, D. A. Boas, J. S. George, and A. J. Durkin, "Topics in biomedical optics: introduction," Appl. Opt. 42, 2869-3329 (2003).
17. W. Cai, M. Xu, and R. R. Alfano, "Three dimensional radiative transfer tomography for turbid media," IEEE J. Sel. Top. Quantum Electron. 9, 189-198 (2003).

18. L. Wang, P. P. Ho, C. Liu, G. Zhang, and R. R. Alfano, "Ballistic 2-D imaging through scattering walls using an ultrafast optical Kerr gate," Science 253, 769-771 (1991).

19. R. R. Alfano, X. Liang, L. Wang, and P. Ho, "Time-resolved imaging of translucent droplets in highly scattering media," Science 264, 1913-1914 (1994).

20. W. Cai, M. Lax, and R. R. Alfano, "Analytical solution of the elastic Boltzmann transport equation in an infinite uniform medium using cumulant expansion," J. Phys. Chem. B 104, 3996-4000 (2000).

21. W. Cai, M. Lax, and R. R. Alfano, "Analytical solution of the polarized photon transport equation in an infinite uniform medium using cumulant expansion," Phys. Rev. E 63, 016606 (2000).

22. M. Xu, W. Cai, M. Lax, and R. R. Alfano, "Photon migration in turbid media using a cumulant approximation to radiative transfer," Phys. Rev. E 65, 066609 (2002).

23. F. Natterer, The Mathematics of Computerized Tomography (Wiley, New York, 1986).

24. A. H. Gandjbakhche, G. H. Weiss, R. F. Bonner, and R. Nossal, "Photon path-length distributions for transmission through optically turbid slabs," Phys. Rev. E 48, 810-818 (1993).

25. A. H. Gandjbakhche, V. Chernomordik, J. C. Hebden, and R. Nossal, "Time-dependent contrast functions for quantitative imaging in time-resolved transillumination experiments," Appl. Opt. 37, 1973-1981 (1998).

26. P. Comon, "Independent component analysis—a new concept?" Signal Process. 36, 287-314 (1994).

27. A. J. Bell, "Information theory, independent component analysis, and applications," in Unsupervised Adaptive Filtering, Vol. I, S. Haykin, ed. (Wiley, New York, 2000), pp. 237-264.

28. D. Nuzillard and J.-M. Nuzillard, "Application of blind source separation to 1-D and 2-D nuclear magnetic resonance spectroscopy," IEEE Signal Process. Lett. 5, 209-211 (1998).

29. R. Vigário, J. Särelä, V. Jousmäki, M. Hämäläinen, and E. Oja, "Independent component approach to the analysis of EEG and MEG recordings," IEEE Trans. Biomed. Eng. 47, 589-593 (2000).

30. A. Hyvärinen, J. Karhunen, and E. Oja, Independent Component Analysis (Wiley, New York, 2001).

31. P. M. Morse and H. Feshbach, Methods of Theoretical Physics (McGraw-Hill, New York, 1953), Vols. I and II.

32. M. Xu, W. Cai, M. Lax, and R. R. Alfano, "A photon transport forward model for imaging in turbid media," Opt. Lett. 26, 1066-1068 (2001).

33. S. Chandrasekhar, Radiative Transfer (Dover, New York, 1960).

34. M. Lax, V. Nayaramamurti, and R. C. Fulton, "Classical diffusion photon transport in a slab," in Laser Optics of Condensed Matter, J. L. Birman, H. Z. Cummins, and A. A. Kaplyanskii, eds. (Plenum, New York, 1987), pp. 229-237.

35. J. X. Zhu, D. J. Pine, and D. A. Weitz, "Internal reflection of diffusive light in random media," Phys. Rev. A 44, 3948-3959 (1991).

36. R. C. Haskell, L. O. Svaasand, T.-T. Tsay, T.-C. Feng, M. S. McAdams, and B. J. Tromber, "Boundary conditions for the diffusion equation in radiative transfer," J. Opt. Soc. Am. A 11, 2727-2741 (1994).

37. S. Roberts and R. Everson, eds., Independent Component Analysis: Principles and Practice (Cambridge U. Press, Cambridge, UK, 2001).

38. J.-F. Cardoso, "Blind signal separation: statistical principles," Proc. IEEE 86, 2009-2025 (1998).

39. M. V. Klein, Optics (Wiley, New York, 1970).

40. H. Heusmann, J. Kölzer, and G. Mitic, "Characterization of female breasts in vivo by time resolved and spectroscopic mea- 
surements in near infrared spectroscopy," J. Biomed. Opt. 1, 425-434 (1996).

41. H. J. van Staveren, C. J. M. Moes, J. van Marle, S. A. Prahl, and M. J. C. van Gemert, "Light scattering in intralipid-10\% in the wavelength range of 400-1100 nm," Appl. Opt. 30, 45074514 (1991).
42. D. J. Hall, J. C. Hebden, and D. T. Delpy, "Imaging very-lowcontrast objects in breastlike scattering media with a timeresolved method," Appl. Opt. 36, 7270-7276 (1997).

43. Q. Fu, F. Seier, S. K. Gayen, and R. R. Alfano, "High-averagepower kilohertz-repetition-rate sub-100-fs Ti:sapphire amplifier system," Opt. Lett. 22, 712-714 (1997). 